\title{
BMJ Open Infant outcomes after exposure to Tdap vaccine in pregnancy: an observational study
}

\author{
Tony Walls, ${ }^{1}$ Patricia Graham, ${ }^{1,2}$ Helen Petousis-Harris, ${ }^{3}$ Linda Hill, ${ }^{4}$ \\ Nicola Austin ${ }^{1,2}$
}

To cite: Walls T, Graham P, Petousis-Harris $\mathrm{H}$, et al. Infant outcomes after exposure to Tdap vaccine in pregnancy: an observational study. BMJ Open 2016;6: e009536. doi:10.1136/ bmjopen-2015-009536

- Prepublication history for this paper is available online. To view these files please visit the journal online (http://dx.doi.org/10.1136/ bmjopen-2015-009536).

Received 30 July 2015 Revised 4 December 2015 Accepted 7 December 2015

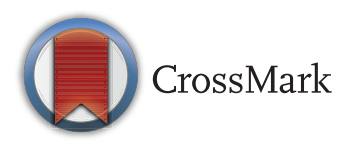

\footnotetext{
${ }^{1}$ Department of Paediatrics, University of Otago, Christchurch, New Zealand ${ }^{2}$ Canterbury District Health Board, Christchurch, New Zealand ${ }^{3}$ Department of General Practice and Primary Health Care, University of Auckland, Auckland, New Zealand ${ }^{4}$ Centre for Postgraduate Nursing Studies, University of Otago, Christchurch, New Zealand
}

Correspondence to Dr Tony Walls; tony.walls@otago.ac.nz

\section{ABSTRACT}

Objective: Pertussis vaccination during pregnancy has recently been recommended in both the USA and UK to prevent pertussis infection in infants. While there are no apparent safety concerns about the administration of Tdap vaccine during pregnancy, there is only limited safety data available. We aimed to closely monitor infants exposed to Tdap during pregnancy to look for any adverse outcomes that may be attributable to the vaccine.

Design: This was a prospective observational study, collecting information to evaluate the safety of Tdap vaccine for infants exposed during pregnancy. Infants were followed for between 6 and 12 months after birth, with $84 \%$ completing 12 months of follow-up. Information was obtained from objective sources including routine health visits and vaccination records wherever possible, as well as frequent parental reports. Setting: The Canterbury region of New Zealand.

Patients: A cohort of 403 infants whose mothers had received Tdap vaccine.

Main outcome measures: Gestational age at birth, growth parameters, congenital anomalies, immunisation status and timeliness of immunisation, development of pertussis infection.

Results: There were no significant differences in birth weight, gestational age at birth, congenital anomalies or infant growth as compared with baseline population data. Infants of mothers who had received the vaccine were more likely to receive their vaccinations on time during infancy. No cases of pertussis occurred in this cohort despite high rates of disease in the community. We have not found any adverse events attributable to vaccine exposure.

Conclusions: These data add to the growing pool of evidence that the administration of Tdap vaccine during pregnancy is an appropriate strategy for reducing the burden of pertussis in infants.

Clinical trial registration: Australia New Zealand Clinical Trials Registry ACTRN12613001045707.

\section{INTRODUCTION}

Young infants are the ones at the greatest risk of complications from pertussis should they

\section{Strengths and limitations of this study}

- We had frequent contact with parents to determine adverse events using multiple modes of communication.

- Wherever possible measures of infant health outcomes were obtained from objective source data.

- Our study population is unlikely to be truly representative of the overall population due to our recruitment methods.

- We have not included a comparison group of infants not exposed to the vaccine during pregnancy.

aquire it. In the absence of maternal immunisation, infants younger than 2 months of age are generally not protected from pertussis until they can be vaccinated as little or no protective antibodies are transferred across the placenta. This is due to the relatively low antibody titres in most pregnant women, as active placental transfer of maternal pertussisspecific antibodies occurs in general leading to higher levels of pertussis-specific IgG in infants than the mother. ${ }^{1}$ In 2012, the US Advisory Committee on Immunization Practices (ACIP) recommended that acellular pertussis vaccine (Tdap) be given to any person likely to be in contact with young infants under the age of 12 months, including pregnant women regardless of previous Tdap vaccination. ${ }^{2}$ Similarly in 2012, the Joint Committee on Vaccination and Immunisation in the UK agreed to the introduction of a programme of immunisation of women with Tdap in the later stages of pregnancy. ${ }^{3}$ Administering the vaccine to pregnant women is advised because it not only protects the mother from pertussis but also induces antibodies that are passed to the infant prior to birth which provide protection for the first weeks of life. ${ }^{2}$ ACIP acknowledged that the safety of Tdap immunisation during pregnancy has not been systematically 
studied, with the only data available coming from small studies, postmarketing surveillance and the US Vaccine Adverse Event Reporting System (VAERS). ${ }^{4}$ They concluded that "available data from these studies did not suggest any elevated frequency or unusual patterns of adverse events in pregnant women who received Tdap and that the few serious adverse events reported were unlikely to have been caused by the vaccine."

In a small phase 1-2 randomised controlled trial (RCT), Munoz et al $\tilde{l}$ demonstrated that Tdap vaccine can safely be given to women during pregnancy without significant adverse events in the mother or the infant. The study included 33 infants exposed in utero to Tdap vaccine. Given the difficulties associated with clinical trials of vaccines administered during pregnancy, there are unlikely to be any adequately powered RCTs to assess the safety of Tdap vaccine. We are hence reliant on the gradual accumulation of data from small trials and observational studies.

The aim of this study was to describe any adverse events following immunisation related to the administration of Tdap vaccine during pregnancy with a focus on infant outcomes.

\section{PATIENTS AND METHODS}

The New Zealand (NZ) immunisation schedule includes a three dose infant schedule of acellular pertussis containing vaccine given at 6 weeks, 3 and 5 months of age with boosters at 4 and 11 years of age. In response to a recent pertussis epidemic in NZ, in 2012 the Ministry of Health introduced Tdap vaccine during pregnancy for women between 28 and 38 weeks of gestation. The funded vaccine was Boostrix (GSK). In NZ all immunisations are provided at no cost to parents through general practitioners (GP) and are recorded through the National Immunisation Register (NIR). In the period October to December 2014, the national immunisation coverage rates were $94 \%$ for both infants aged 8 and 24 months. ${ }^{6}$

The Safety Monitoring of Adverse Reactions to Tdap Vaccine in Pregnancy (SMART VIP) study is a prospective observational cohort study carried out in the Canterbury region of NZ (birth cohort approximately 6000 per annum). It forms part of a larger overall national study looking at adverse events following Tdap vaccination during pregnancy and specifically focuses on longitudinal infant outcomes.

Women were included in the study if they had received Tdap vaccine between 28 and 38 weeks gestation. They had to have been compliant with routine antenatal care, including at least one ultrasound scan early in pregnancy. This was to ensure that any major congenital anomalies identified early in pregnancy were not later attributed to vaccination. We excluded women whose fetus had congenital anomalies, severe structural and/or chromosomal abnormalities identified during prenatal screening.
Women completed a consent form with their GP prior to administration of Tdap asking if they were happy to be contacted by the study team. GP claims for funding reimbursements were used to identify women who had received the vaccine and those who consented were contacted by telephone within 2 weeks of administration. Infant follow-up was planned through to 1 year of age. The first enrolment was in September 2012 and infant follow-up went through until November 2014.

The study was allocated a Universal Trial Number (U1111-1148-0718) on the 16 September 2103 and registered with Australia New Zealand Clinical Trials Registry (ACTRN12613001045707) on the 19 September 2013.

Funding was by an unrestricted grant from the Canterbury District Health Board.

Information on pregnancy and infant outcomes were collected from maternal reporting and confirmed through a variety of objective sources including birth records, notes from the lead maternity carer (LMC; principal healthcare professional responsible for the infant until 6 weeks of age) and the routine infant 6-week check at the GP. These included any adverse events in women during pregnancy, infant gestational age at birth, birth weight, length and head circumference, congenital anomalies, and any medically attended events for the infants following birth.

Gestational age was taken from the last menstrual period and where this was not available, early pregnancy ultrasound was used to estimate this. Preterm birth was defined as any infant delivered before 37 weeks gestation and infants born $<32$ weeks gestation were defined as very preterm. Low birth weight was defined as $<2500 \mathrm{~g}$ with very low birth weight $<1500 \mathrm{~g}$ irrespective of gestational age at birth. The Brighton Collaboration consensus list of terms and concept definitions of key events for monitoring immunisation in pregnancy was used to categorise medical events of significance and congenital anomalies. ${ }^{7}$

Infant immunisation data were obtained from the $\mathrm{NIR}^{6}$ and we documented both the receipt of vaccine and the timeliness of vaccination. On-time immunisation was defined as an infant receiving their vaccine within 1 month of the recommended date. Infant growth parameters were obtained from routine visits to community well-child provider (Plunket Nurse) clinics up to the visit at around 5-7 months.

All of these services are provided at no charge to families as part of routine infant healthcare. Z-scores were used for all growth measurements where appropriate using WHO growth charts.

At monthly intervals, a questionnaire was sent to mothers via mail, email or sms asking if the infants had been in close contact with anyone with proven pertussis infection, or an unexplained cough lasting $>2$ weeks, or if the child had a persistent cough lasting $>10$ days. At least three attempts were made on each occasion to contact parents, using multiple contact methods to ensure infants had complete follow-up. A clinical review 
was arranged for any child with significant contact with someone with pertussis or symptoms consistent with pertussis. Details of any medical assessments required by children and not done by study staff were obtained from hospital or GP records.

Significant or medically attended adverse events in either the mother or their infants were reported to the Centre for Adverse Reactions Monitoring, at the NZ Pharmacovigilance Centre, University of Otago.

This is a descriptive analysis and outcomes are presented in numbers and as percentages. Comparison was made for the rates and timeliness of immunisation for infants between the study population and the total infant population of the Canterbury region during the study period using risk ratios.

\section{RESULTS}

We were notified of 1211 women who had received Tdap vaccine during pregnancy and recruited 470 . The reasons for not participating are listed in figure 1. The mean age of women at the time of Tdap vaccination was

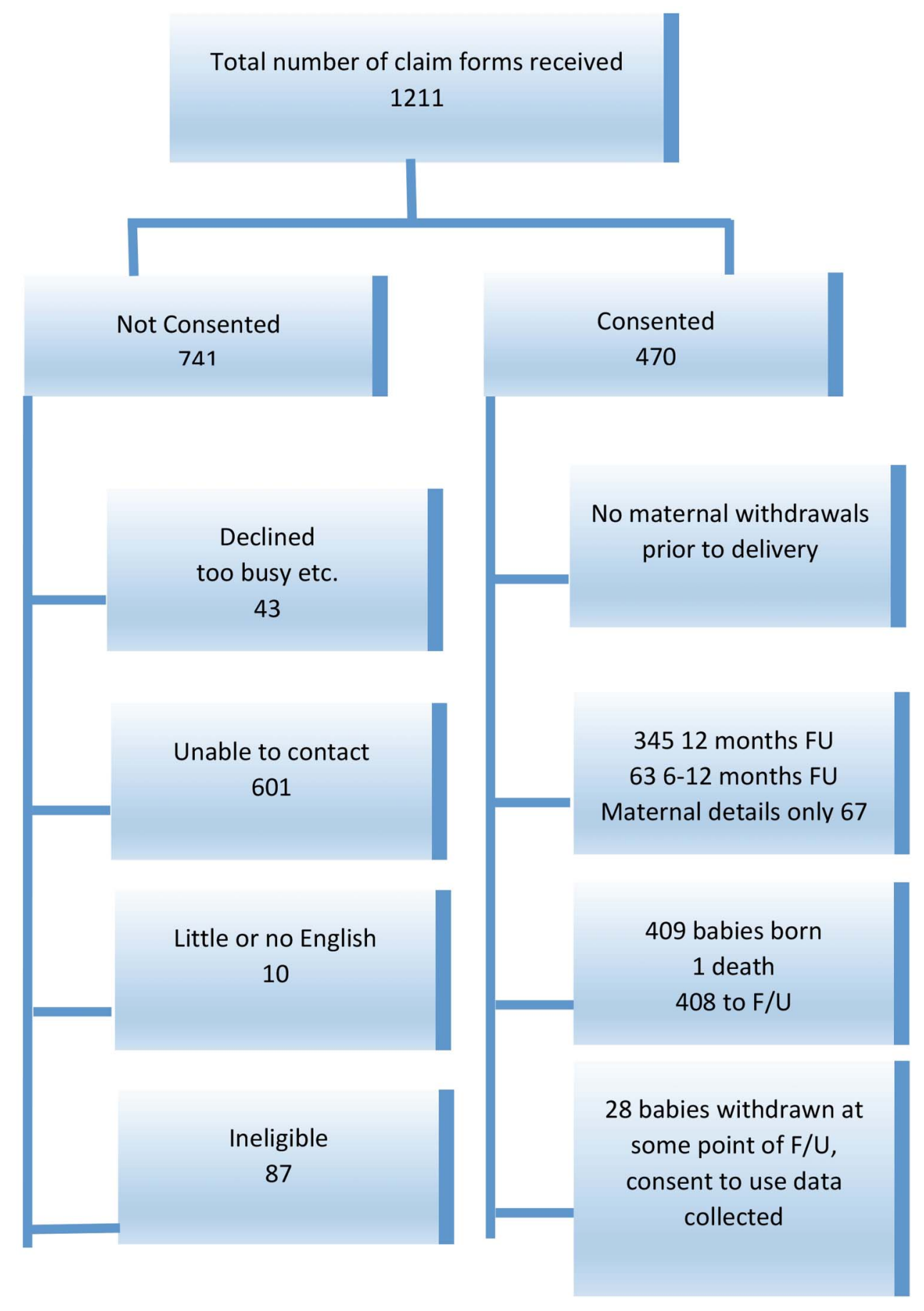

Figure 1 Study enrolment (FU, follow-up). 
32 years (range $15-45$ years) and the average gestational age was 33.9 weeks. Eighty-six per cent of women were NZ European with $4 \%$ Maori, and $8 \%$ Asian. Sixty-two women also received trivalent influenza vaccine during their pregnancy, often given at the same time at Tdap vaccine.

Follow-up data were obtained from 403 women on 408 infants (403 singleton infants, 6 sets of twins and 1 stillbirth), 345 of these through to 12 months of age and 63 through to 6-12 months of age. We elected to include infants followed through to at least 6 months in the analysis as we felt that most medically significant events relating to maternal vaccination would have presented by this time. In 67 cases, maternal data only were available and these were not included in the analysis. There were 28 infants who were withdrawn from the study.

Three hundred and eighty-five of the infants followed up delivered at term (94\%). The average gestational age at delivery was 39.2 weeks (range 33-42 weeks). There were six sets of twins ( 3 sets born at term, 2 sets born at 36 weeks and 1 at 33 weeks gestation). Only $23(6 \%)$ infants were born at less than 37 weeks gestation. The mean birth weight was $3490 \mathrm{~g}$ with only one infant weighing $<1500 \mathrm{~g}$ and eight infants between 1500 and $2500 \mathrm{~g}$.

Ten infants $(2.5 \%)$ were identified as having medical events of significance or congenital anomalies as listed in box 1. One infant was stillborn $(0.2 \%)$ and despite a postmortem examination (there were no congenital abnormalities identified) the reason for this is unknown. The mother had a history of previous stillbirth.

A total of 303 infants completed their 6-week check and 278 completed their 5-month check. Figure 2 shows the z-scores for weight at birth, at the 6 -week check and at between 5 and 7 months of age, each of which is normally distributed.

Only nine infants had contact with a confirmed case of pertussis during the follow-up period. Sixty-seven infants were household contacts of a person with a prolonged cough illness and 64 infants were reported to have had a cough lasting $>10$ days themselves. None of these infants were subsequently diagnosed with pertussis. Only nine infants were admitted to hospital during the

Box 1 Medical events of significance and congenital anomalies in infants exposed to Tdap during pregnancy

Gastroschisis
Malrotation of bowel
Benign neonatal myoclonus
Hypospadias
Subluxation right hip
Perforated bowel
Pyloric stenosis
Hip dysplasia
Right hand anomaly
Laryngomalacia

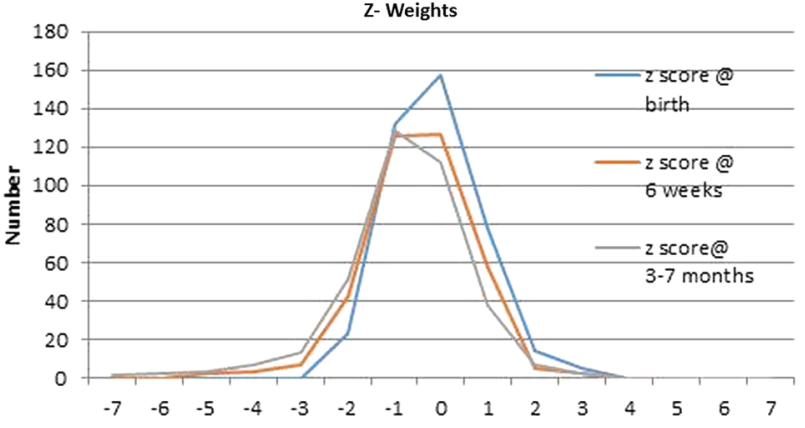

Figure 2 Z-scores for infant weights at birth, 6 weeks and 5-7 months of age.

follow-up period. Three of these had respiratory tract illness, one of whom had proven influenza infection and all three tested negative for pertussis.

The proportions of infants in the SMART VIP cohort receiving their immunisations on time were $97.8 \%$, $98.5 \%$ and $94.2 \%$ at 6 weeks, 3 and 5 months, respectively. For each vaccination event, this was significantly better than the overall Canterbury infant cohort during the same time period (table 1).

\section{DISCUSSION}

Despite its widespread use in the USA and the UK, there is still limited safety data on the use of Tdap vaccine during pregnancy. This is the largest study to date investigating individual health outcomes and prospectively monitoring for adverse events in infants whose mothers had received Tdap vaccine during pregnancy.

This study looked at infant outcomes and found no evidence of increased adverse events for the infant who has been exposed in utero to Tdap vaccine when compared with the baseline population rates. Birth outcomes, growth parameters and the rates of congenital anomalies were very similar to the baseline rates in NZ. The NZ Ministry of Health report on maternity clinical indicators $^{8}$ reported $8 \%$ of all live infants were born between 32 and 36 weeks gestation across the Canterbury region. This was higher than the $6 \%$ rate of prematurity in our cohort.

The NZ birth defects registry ${ }^{9}$ listed the rates of congenital anomalies ranging between 4000 and 6000 per 100000 hospital births (between 4\% and 6\%) for the years 2000-2012. In our cohort, the rates were well below this at $2.7 \%$. These comparisons must be made with caution given the selective nature of our study population. In particular, it is likely that rates of congenital anomalies in our study population would be lower than comparative data given our cohort did not include any infants born before 33 weeks gestation. However, it is reassuring that there is no signal suggestive of potential adverse outcomes from these data.

The only infant outcome that was significantly different in our cohort from the Canterbury infant population was the timeliness of infant immunisations. On-time 
Table 1 Timeliness of vaccinations in the Safety Monitoring of Adverse Reactions to Tdap Vaccine in Pregnancy (SMART VIP) cohort and the non-study Canterbury infant population

\begin{tabular}{|c|c|c|c|c|c|c|}
\hline & Total (n) & On-time (n) & Overdue (n) & Overdue (\%) & Risk ratio $(95 \% \mathrm{Cl})$ & $p$ Value \\
\hline \multicolumn{7}{|l|}{6 weeks } \\
\hline Non-study & 5776 & 5374 & 402 & 7.1 & 1 & \\
\hline SMART VIP cohort & 403 & 394 & 9 & 2.2 & 0.32 (0.17 to 0.62$)$ & $<0.0001$ \\
\hline \multicolumn{7}{|l|}{3 months } \\
\hline Non-study & 5781 & 5082 & 699 & 12.1 & 1 & \\
\hline SMART VIP cohort & 398 & 392 & 6 & 1.5 & $0.12(0.06$ to 0.28$)$ & $<0.0001$ \\
\hline \multicolumn{7}{|l|}{5 months } \\
\hline Non-study & 5781 & 4764 & 1017 & 17.6 & 1 & \\
\hline SMART VIP cohort & 398 & 375 & 23 & 5.8 & $0.33(0.22$ to 0.49$)$ & $<0.0001$ \\
\hline
\end{tabular}

immunisation of infants is one of the key public health interventions recommended to reduce the risk of pertussis in infants during an epidemic. Delayed immunisation has repeatedly been associated with higher rates of pertussis in infants and hospitalisation with pertussis. ${ }^{10}$

The infants born to mothers in our cohort who received Tdap vaccine were more likely to receive their vaccines on time when compared with the Canterbury population rates. One possible explanation for this is that women who chose to be vaccinated, and agreed to participate in the study, were more aware of the ongoing pertussis epidemic in the community and thus more likely to choose to vaccinate their infants on time. In $\mathrm{NZ}$, most of the LMCs are midwives who are not able to administer vaccines. If women wish to be vaccinated they had to attend their GP, something that is not a routine part of antenatal care. This additional GP visit will have provided an opportunity to discuss the benefits of immunisation for both themselves and their infants with a medical practitioner. The introduction of the vaccine may have inadvertently led to more contact of expectant mothers with their GPs and more discussion about immunisation.

In general, there are no theoretical safety concerns with administering subunit vaccines to pregnant women. Some vaccines, such as tetanus, are used widely in this group without evidence of significant adverse events in the mother or infant. ${ }^{11}$ The timing of maternal immunisation is also an important factor for vaccine safety. When Tdap vaccine is given between 28 and 38 weeks gestation, the risks of congenital anomalies is likely to be low given the period of major fetal embryogenesis has been completed by that gestation. However, compared with vaccines administered outside of pregnancy, there is often limited safety data available because of the lack of large RCTs to evaluate these. In the absence of data from RCTs accumulation of information from welldesigned observational studies becomes increasingly important. This study contributes to our knowledge of the safety of Tdap vaccine given during pregnancy and we have agreed to include the study data in an international database on pregnancy exposures to Tdap.

Recent estimates of vaccine effectiveness suggest that vaccinating women late in the third trimester of pregnancy is $91 \%$ protective against the infant developing pertussis in the first 2 months of life. ${ }^{12}{ }^{13}$ None of the infants in our cohort developed pertussis. This was an observational study involving only infants exposed to vaccine, so we are unable to draw any conclusions about vaccine efficacy. However, given the high rates of pertussis in the community through the duration of the study, it is very reassuring that there were no cases of disease. This would be consistent with the estimated effectiveness of the vaccine.

One of the strengths of this study was that by frequent contact with parents using multiple modes of communication, we had high numbers of infants completing follow-up. We chose to use objective data measures from routine sources such as the NIR to provide optimal data quality.

While we were able to obtain data on a large number of women and infants, the observational design however meant that our sample population is not truly representative of the overall population in the region. For example, our cohort had fewer Maori and Pacific Island women than the rest of Canterbury, and in both these ethnic groups, rates of pertussis infection has been shown to be significantly higher than in NZ Europeans. ${ }^{14}$ We also need to emphasise that the women approached to take part in this study were a subgroup of those vaccinated who had given consent to be contacted by the research team. Owing to resource constraints, we were unable to contact all of these women, and unfortunately we have no data to compare the vaccinated women who did not participate in the study with our study population.

A small proportion of women in this study also received seasonal influenza vaccine, often given simultaneously with Tdap vaccine. Given the observational nature of the study, we were unable to control for this; however, there were no statistically significant differences in outcomes for women who received both vaccines as compared with those who did not receive influenza vaccine (data not shown).

Finally, while this is the largest study to date with detailed observations of infants exposed to Tdap vaccine during pregnancy, the number of participants is still too small to detect less common adverse events attributable to the vaccine. 


\section{CONCLUSIONS}

Administering Tdap vaccine during pregnancy has previously been demonstrated to provide protection for infants from pertussis during epidemics. We have shown no difference in infant outcomes in those who have been exposed to the vaccine in pregnancy when compared with the overall population. This provides further evidence to back up recommendations for vaccinating pregnant women with Tdap to prevent pertussis in their infants. However, the data on vaccine safety remain limited, and the need for ongoing surveillance and reporting of adverse events relating to Tdap vaccination during pregnancy remains.

Twitter Follow Helen Petousis-Harris at @PetousisH

Acknowledgements The authors thank Dr Jonathan Williman for advice on the statistical analysis. They also thank the CDHB for their financial support for this research.

Contributors TW conceptualised and designed the study, drafted the manuscript and approved the final manuscript as submitted. PG designed the data collection tool and collected and collated most of the data. She helped with drafting the manuscript and approved the final version. HP-H was involved with the study concept development and design, as well as the initial funding application. She helped with drafting the manuscript and approved the final manuscript as submitted. LH was involved with data collection and analysis of data relating to immunisation status of the infants. She helped with drafting the manuscript and approved the final manuscript as submitted. NA was involved with study planning and design as well as the initial funding application. She helped with drafting the manuscript and approved the final version.

\section{Funding Canterbury District Health Board.}

Competing interests TW has done investigator-led studies funded by GSK. HP-H has done investigator-led studies funded by GSK, CSL and Sanofi.

Patient consent Obtained.

Ethics approval Upper South A Regional Ethics Committee-approval URA/ 12/EXP/021.

Provenance and peer review Not commissioned; externally peer reviewed.

Data sharing statement No additional data are available.

Open Access This is an Open Access article distributed in accordance with the Creative Commons Attribution Non Commercial (CC BY-NC 4.0) license, which permits others to distribute, remix, adapt, build upon this work non- commercially, and license their derivative works on different terms, provided the original work is properly cited and the use is non-commercial. See: http:// creativecommons.org/licenses/by-nc/4.0/

\section{REFERENCES}

1. Healy CM, Munoz FM, Rench MA, et al. Prevalence of pertussis antibodies in maternal delivery, cord, and infant serum. J Infect Dis 2004;190:335-40.

2. Centers for Disease Control and Prevention CDC. Updated recommendations for use of tetanus toxoid, reduced diphtheria toxoid, and acellular pertussis vaccine (Tdap) in pregnant womenAdvisory Committee on Immunization Practices (ACIP), 2012 MMWR Morb Mortal Wkly Rep 2013;62:131-5.

3. JCVI meeting on pertussis immunisation: August 2012. https://www. gov.uk/government/groups/joint-committee-on-vaccination-andimmunisation

4. Zheteyeva YA, Moro PL, Tepper NK, et al. Adverse event reports after tetanus toxoid, reduced diphtheria toxoid, and acellular pertussis vaccines in pregnant women. Am J Obstet Gynecol 2012;207:59.e1-7.

5. Munoz FM, Bond $\mathrm{NH}$, Maccato $\mathrm{M}$, et al. Safety and immunogenicity of tetanus diphtheria and acellular pertussis (Tdap) immunization during pregnancy in mothers and infants: a randomized clinical trial. JAMA 2014;311:1760-9.

6. National Immunisation Register Publications. http://www.health.govt. nz/our-work/preventative-health-wellness/immunisation/nationalimmunisation-register/national-immunisation-register-publications (accessed 9 Mar 2015).

7. Munoz FM, Eckert LO, Katz MA, et al. Key terms for the assessment of the safety of vaccines in pregnancy: results of a global consultative process to initiate harmonization of adverse event definitions. Vaccine 2015;33:6441-52.

8. New Zealand Maternity Clinical Indicators. http://www.health.govt.nz/ publication/new-zealand-maternity-clinical-indicators-2012 (accessed 9 Mar 2015)

9. New Zealand Birth Defects Registry. http://nzbdr.ac.nz (accessed 9 Mar 2015).

10. Grant CC, Roberts M, Scragg R, et al. Delayed immunisation and risk of pertussis in infants: unmatched case-control study. BMJ 2003;326:852-3.

11. Lindsey B, Kampmann B, Jones C. Maternal immunization as a strategy to decrease susceptibility to infection in newborn infants. Curr Opin Infect Dis 2013;26:248-53.

12. Amirthalingam $\mathrm{G}$, Andrews $\mathrm{N}$, Campbell $\mathrm{H}$, et al. Effectiveness of maternal pertussis vaccination in England: an observational study. Lancet 2014;384:1521-8.

13. Dabrera G, Amirthalingam G, Andrews N, et al. A case-control study to estimate the effectiveness of maternal pertussis vaccination in protecting newborn infants in England and wales, 2012-2013. Clin Infect Dis 2015;60:333-7.

14. Somerville RL, Grant CC, Grimwood K, et al. Infants hospitalised with pertussis: estimating the true disease burden. J Paediatr Child Health 2007;43:617-22. 